\title{
Endoscopic Endonasal Sinus Stent Placement as a Salvage Technique for Drainage of a Petrous Abscess
}

\author{
Gilbert Cadena, $M D^{1 *}$, Benjamin Bitner, BS ${ }^{2}$, Katelynn Tran ${ }^{1}$, Naveen Bhandarkar, $M D^{2}$ and Frank PK \\ Hsu, MD, PhD ${ }^{1}$
}

${ }^{1}$ Department of Neurological Surgery, University of California, Irvine, USA

${ }^{2}$ Department of Otolaryngology - Head and Neck Surgery, University of California, Irvine, USA

\begin{abstract}
Various cranial approaches exist to address lesions of the petrous apex. Many of these involve skull base approaches with varying degrees of surgical morbidity. We present a unique case of a recurrent petrous abscess that was treated with an endoscopic skull base approach with placement of a permanent stent commonly used for sinus surgery. To our knowledge this is the first report to repurpose this type of stent and employ it long-term (3+ years) without any significant side-effects.
\end{abstract}

Keywords

Expanded endonasal approach, Epidermoid cyst, Endoscopic stent, Endoscopic skull base surgery, Transcavernous sinus approach

\section{Introduction}

Epidermoid cysts represent the third most common entity encountered in the cerebellopontine (CP) angle. Uncontrolled, they may grow to encompass the adjacent petrous apex and middle fossa, creating a formidable surgical lesion. While various open trans-cranial skull base approaches have been used to address these lesions, surgical morbidity can be significant, and cure is difficult due to the intimate association with critical neurovascular structures. Morbidity is increased when these lesions become secondarily infected. Endoscopic skull base approaches exploit the natural sinonasal corridor as a minimally invasive route to access lesions of the petrous apex and middle fossa [15]. Though various reports describe endoscopic-assisted techniques to resect epidermoid cysts [6-9], we present the utility of anendoscopic endonasal approach with placement of apermanent Rains frontal sinus stent as a unique and viable surgical technique for drainage of a complex petrous abscess with brainstem compression.

\section{Case Description}

A 35-year-old woman presented to an outside facility in 2005 with hearing loss and cranial neuropathy caused by a left $C P$ angle epidermoid. She underwent two extended translabyrinthine approaches before presenting to our institution with recurrence in 2014. At our institution, she underwent a middle fossa approach for tumor debulking and brainstem decompression. Postoperatively, she developed a wound infection and CSF leak that required re-exploration, temporary CSF diversion, and an extended course of parenteral antibiotics. One month later, she presented with an intracranial abscess filling the resection cavity, CP angle, prepontine cistern, and petrous apex with mass effect on the brainstem (Figure 1). Given her history of multiple prior craniotomies on the dominant hemisphere with subsequent wound breakdown and CSF leak, an endoscopic endonasal approach was chosen as the most direct route to approach the petrous portion of the abscess. A frontal sinus stent commonly used in sinus surgery was placed endoscopically for drainage. The stent was removed in a delayed fashion. The patient made a slow, but near-complete recovery over the next several months. However, she slowly developed worsening headaches and left CN VI and VII palsies. Imaging demonstrated slow recurrence of the epidermoid cyst after the stent had been removed. She was taken back to the operating room

*Corresponding author: Gilbert Cadena, MD, Department of Neurological Surgery, University of California, Irvine, 200 South Manchester Ave, Suite 210, Orange, CA 92868, USA, Tel: 714456-7495, Fax: 724-456-8212

Accepted: November 09, 2019

Published online: November 11, 2019

Citation: Cadena G, Bitner B, Tran K, et al. (2019) Endoscopic Endonasal Sinus Stent Placement as a Salvage Technique for Drainage of a Petrous Abscess. J Head Neck Surg 2(1):45-48 

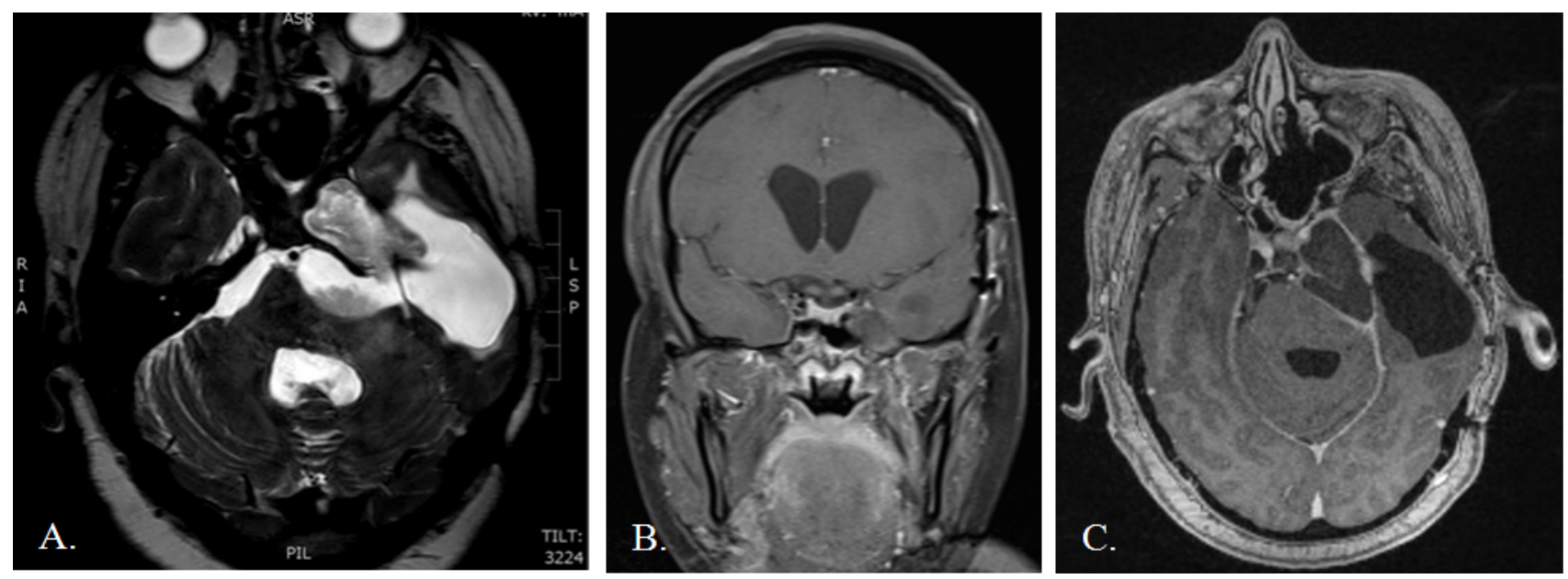

Figure 1: Preoperative axial T2 weighted MRI demonstrates a left petrous abscess with extension to the prepontine cistern with compression of the pons and erosion of the left petrous apex (A). Preoperative coronal (B) and axial (C) post-contrast MRI shows a left petrous abscess displacing the left internal carotid artery medially.
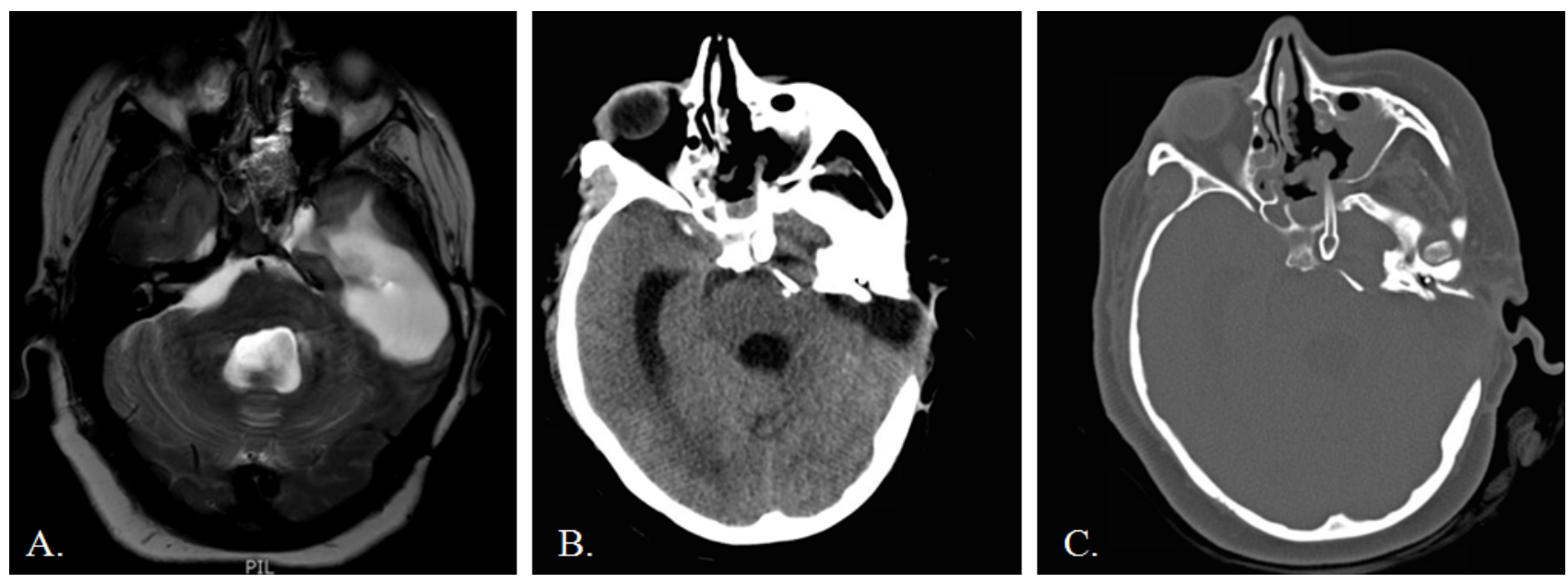

Figure 2: Immediate postoperative axial T2 weighted MRI demonstrates resolution of the large epidermoid cyst and brainstem decompression (A). Postoperative axial CT scans show placement of a Rains stent into the region of the left petrous apex (B,C).

for re-exploration and a redo endoscopic drainage. We left apermanent stent to maintain a path for continuous drainage. Immediate and delayed postoperative imaging showed resolution of the abscess with good brainstem decompression (Figure 2). Her seventh nerve palsy improved immediately after surgery. Her left sixth nerve palsy has remained stable. She has had no complications as a result of stent placement three years out from the last operation.

\section{Surgical Technique}

\section{Initial endonasal approach}

A $4 \mathrm{~mm}$ rigid endoscope (Karl Storz, Tuttlingen, Germany) was introduced into the nose. A wide sphenoidotomy was created and the left middle turbinate was resected. A pedicled nasoseptal flap was harvested, according the technique of Hadad and Bassagasteguy [10]. The petrous abscess had displaced the cavernous carotid medially, leaving a lateral corridor via the sphenoid sinus that lead to the abscess cavity. Using intraoperative navigation, we identified the thin bone overlying the petrous apex. The skull base was carefully drilled using a $3 \mathrm{~mm}$ course diamond bit. After dural opening, purulent contents were immediately visualized and evacuated under pressure. Several pieces of keratin debris were irrigated from the cavity as well. There was no evidence of CSF leak from the cavity. A Rains frontal sinus stent was placed in reverse orientation into the fluid collection with a suture attached to the end for easy retrieval. Finally, a pedicled nasoseptal flap was placed over the skull base defect.

\section{Subsequent endonasal approach}

Upon entering the nasal cavity for the second time, intraoperative navigation was used to visualize an area of scar tissue in the prior stent's vicinity. The scar was opened sharply to gain access to the reaccumulated petrous abscess. A small amount of low-pressure venous bleeding was encountered, followed by egress of cloudy fluid under moderate pressure as we opened the capsule. Small pieces of keratin debris were mixed with the fluid contents, con- 


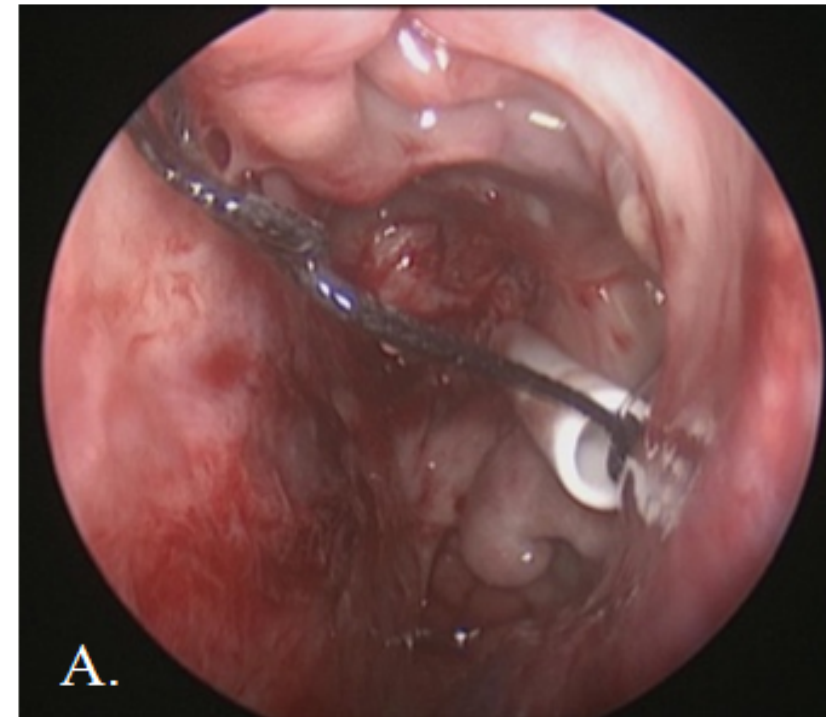

Figure 3: Endoscopic view of a Rains silastic stent providing a drainage pathway for petrous abscess (A).

sistent with remnants of epidermoid and abscess. A frontal sinus stent was gently placed through the opening into the abscess cavity (Figure 3). We copiously irrigated the cavity to ensure stent patency. There was no evidence of CSF leak during the case.

\section{Discussion}

Epidermoid cysts are extra-axial lesions commonly found in the parasellar cisterns and cerebellopontine angle. Symptoms are often the result of local mass effect and occasionally obstructive hydrocephalus. The capsule adheres to adjacent neurovascular structures, making surgical cure challenging. Growth of these lesions is linear and typically slow, but they can often span multiple intracranial compartments. While various open trans-cranial approaches can be employed, each has its own set of risks, including brain retraction, CSF leak, hearing loss, facial nerve injury, cosmetic deformity, and infection. More recently, several groups have reported endoscopic-assisted micro neurosurgical techniques for resection [6-8] to reduce surgical morbidity.

By contrast, lesions that encompass the region of the petrous apex and medial middle fossa may be more amenable to purely endoscopic skull base techniques [11-14]. The natural sinonasal corridor can be exploited as a means of approaching most areas of the skull base using a rigid endoscope, while allowing one to maintain bimanual microsurgical technique. For lesions in the petrous apex, a natural path of drainage can be created and maintained with or without placement of a stent. Expansile lesions often displace the carotid artery and sometimes protrude into the sphenoid sinus, making them amenable to simple endoscopic transsphenoidal approaches. Alternatively, those that lie lateral or inferior to the carotid artery may require additional techniques involving extended transpterygoid approaches $[1,14]$. Each of these approaches presents a unique, minimally invasive route to the petrous apex, but requires thorough familiarity with endoscopic skull base anatomy and the assistance of intraoperative surgical navigation systems.

We describe a unique case of a recurrent CP angle epidermoid cyst that was successfully treated using a purely endoscopic technique. This particular lesion encompassed the petrous apex with extension to the posterior fossa. Because various open techniques previously used lacked durable results, the case was considered a unique surgical challenge. We felt that another craniotomy would pose significant risk to her dominant temporal lobe and risked wound breakdown, potential CSF leak, and postoperative seizures. Extrapolating well-described techniques of cholesterol granuloma stent drainage to this particular circumstance [11-14], we believed that an endonasal approach could achieve a durable result with a lower risk of approach-related morbidity.

Endoscopic stent-drainage of petrous apex lesions is commonly employed to drain cholesterol granulomas with recurrence rates ranging from $4.3 \%$ to nearly $60 \%$, depending upon the use of a stent or vascularized flap to prevent restenosis $[11-13,15,16]$. The particular stents typically used for drainage include silastic stents and t-shaped stents. Though cholesterol granulomas and petrous abscesses have fundamentally different pathophysiology, our goal was to employ a minimally invasive method to provide cyst decompression and prevent recurrence. To our knowledge, our case is one of two reports of a purely endoscopic stent-drainage technique for this type of pathology. Ibrahim, et al. reported the use of a silastic stent for drainage of a recurrent epidermoid cyst in the infratemporal fossa accessed via a transnasal transpterygoid approach [17]. We chose to use a Rains frontal sinus stent, which was originally designed to be used for management of frontal sinus disease requiring drainage [18]. The stent is self-retaining, eliminating the need for suturing and is easier to insert endoscopically compared to other traditional silastic stents. The use of a Rains frontal sinus stent has not been described as a strategy for drainage of petrous abscesses.

Our initial attempt to remove the stent in a delayed fashion failed. The outflow tract quickly obstructed, allowing the abscess to reaccumulate. This was accompanied by cranial nerve palsy and headache. Other authors have advocated use of a vascularized graft to maintain patency of draining ostia; however, these were in cases of simple lesions confined to the petrous apex $[15,16]$. In our case, a pedicled nasoseptal flap was initially placed over the skull base defect to prevent CSF leak. This precluded placement of a vascularized flap during the second operation. Initial postoperative scans demonstrated excellent decompression, providing proof that this technique could be employed successfully for this purpose.

In conclusion, we describe a unique solution to a petrous abscess by employing a fully endoscopic skull base approach with the use of a Rains frontal sinus stent. The challenges of this approach are inherent to the surrounding neurovascular structures of the cavernous sinus and skull base. Given the 
expansile nature of the lesion with carotid displacement, we felt that this was an ideal approach that was direct, remained extra-dural, and avoided brain manipulation. The patient's headaches and facial nerve palsy immediately improved after cyst decompression. The sixth nerve palsy has remained stable for several months. Though more experience is necessary to draw any firm conclusions, endoscopic stent-drainage of petrous apex abscesses is a viable solution that avoids many of the potential morbidities associated with trans-cranial approaches.

\section{Conflict of Interests}

The authors report no financial disclosures or conflict of interest.

\section{References}

1. Kassam AB, Gardner P, Snyderman C, et al. (2005) Expanded endonasal approach: Fully endoscopic, completely transnasal approach to the middle third of the clivus, petrous bone, middle cranial fossa, and infratemporal fossa. Neurosurg Focus 19: E6.

2. de Lara D, Ditzel Filho LF, Prevedello DM, et al. (2014) Endonasal endoscopic approaches to the paramedian skull base. World Neurosurg 82: S121-S129.

3. Kassam AB, Vescan AD, Carrau RL, et al. (2008) Expanded endonasal approach: Vidian canal as a landmark to the petrous internal carotid artery. J Neurosurg 108: 177-183.

4. Snyderman $\mathrm{CH}$, Pant $\mathrm{H}$, Carrau RL, et al. (2009) What are the limits of endoscopic sinus surgery?: The expanded endonasal approach to the skull base. Keio J Med 58: 152-160.

5. Van Gompel JJ, Alikhani P, Tabor MH, et al. (2014) Anterior inferior petrosectomy: Defining the role of endonasal endoscopic techniques for petrous apex approaches. J Neurosurg 120: 13211325.

6. Trivelato FP, Giannetti AV (2008) Endoscope-controlled microneurosurgery to treat middle fossa epidermoid cysts: Technical case report. Neurosurgery 62: 105-106.

7. Ebner FH, Roser F, Thaher F, et al. (2010) Balancing the shortcomings of microscope and endoscope: Endoscope-assisted technique in microsurgical removal of recurrent epidermoid cysts in the posterior fossa. Minim Invasive Neurosurg 53: 218222.
8. Kasliwal MK, Fraser JF, Greenfield JP, et al. (2010) Simultaneous middle fossa arachnoid cyst and ambient cistern epidermoid cyst: Case report and endoscope-assisted microsurgical management. Pediatr Neurosurg 46: 151-154.

9. Kassam AB, Prevedello DM, Carrau RL, et al. (2009) The front door to meckel's cave: An anteromedial corridor via expanded endoscopic endonasal approach- technical considerations and clinical series. Neurosurgery 64: 71-82.

10. Hadad G, Bassagasteguy L, Carrau RL, et al. (2006) A novel reconstructive technique after endoscopic expanded endonasal approaches: Vascular pedicle nasoseptal flap. Laryngoscope 116: 1882-1886.

11. McLaughlin N, Kelly DF, Prevedello DM, et al. (2012) Endoscopic endonasal management of recurrent petrous apex cholesterol granuloma. J Neurol Surg B Skull Base 73: 190-196.

12. Paluzzi A, Gardner P, Fernandez-Miranda JC, et al. (2012) Endoscopic endonasal approach to cholesterol granulomas of the petrous apex: A series of 17 patients: Clinical article. J Neurosurg 116: 792-798.

13. Eytan DF, Kshettry VR, Sindwani R, et al. (2014) Surgical outcomes after endoscopic management of cholesterol granulomas of the petrous apex: A systematic review. Neurosurg Focus 37: E14.

14. Zanation AM, Snyderman CH, Carrau RL, et al. (2009) Endoscopic endonasal surgery for petrous apex lesions. Laryngoscope 119: 19-25.

15. Eisenberg MB, Haddad G, Al-Mefty O (1997) Petrous apex cholesterol granulomas: Evolution and management. J Neurosurg 86: 822-829.

16. Shibao S, Toda M, Tomita T, et al. (2015) Petrous apex cholesterol granuloma: Importance of pedicled nasoseptal flap in addition to silicone T-tube for prevention of occlusion of drainage route in transsphenoidal approach--a technical note. Neurol Med Chir (Tokyo) 55: 351-355.

17. Ibrahim T, Ackerman PD, Welch KC, et al. (2013) Transpterygoid stent placement for management of a recurrent infratemporal fossa epidermoid cyst: A technical note. J Neurol Surg Rep 74: 123-128.

18. Rains BM 3rd (2001) Frontal sinus stenting. Otolaryngol Clin North Am 34: 101-110.

DOI: $10.36959 / 605 / 537$

Copyright: (C) 2019 Cadena G, et al. This is an open-access article distributed under the terms of the Creative Commons Attribution License, which permits unrestricted use, distribution, and reproduction in any medium, provided the original author and source are credited. 